\title{
Transcriptional regulation of genes involved in keratinocyte differentiation by human papillomavirus 16 oncoproteins
}

Eszter Gyöngyösi 1

Anita Szalmás 1

Annamária Ferenczi 1

Szilárd Póliska 2

József Kónya 1

György Veress 1,*

Phone +36-52-255-425

Email veregy@med.unideb.hu

1 Department of Medical Microbiology, Faculty of Medicine, University of Debrecen, Debrecen, Hungary

2 Department of Biochemistry and Molecular Biology, Genomic Medicine and Bioinformatics Core Facility, Faculty of Medicine, University of Debrecen, Debrecen, Hungary

\section{Abstract}

The life cycle of human papillomaviruses (HPVs) is strictly linked to the differentiation of their natural host cells. The HPV E6 and E7 oncoproteins can delay the normal differentiation program of keratinocytes; however, the exact mechanisms responsible for this have not yet been identified. The goal of this study was to investigate the effects of HPV16 oncoproteins on the expression of genes involved in keratinocyte differentiation. Primary human keratinocytes transduced by LXSN (control) retroviruses or virus vectors expressing HPV16 E6, E7 or E6/E7 genes were subjected to gene expression profiling. The results of microarray analysis showed that HPV 
16 E6 and E7 have the capacity to downregulate the expression of several genes involved in keratinocyte differentiation. Quantitative real-time polymerase chain reaction (qRT-PCR) assays were performed to confirm the microarray data. To investigate the effects of the HPV oncoproteins on the promoters of selected keratinocyte differentiation genes, luciferase reporter assays were performed. Our results suggest that the HPV 16 E6 and/or E7 oncogenes are able to downregulate the expression of several genes involved in keratinocyte differentiation (such as desmocollin 1, keratin 4, S100 calcium-binding protein A8 and small proline-rich protein 1A), at least partially by downregulating their promoter activity. This activity of the HPV oncoproteins may have a role in the productive virus life cycle, and also in virus-induced carcinogenesis.

\section{Electronic supplementary material}

The online version of this article (doi:10.1007/s00705-014-2305-y) contains supplementary material, which is available to authorized users.

\section{Introduction}

Certain types of human papillomaviruses (HPVs) (high-risk or oncogenic types, such as HPV 16 and 18) are considered to be the causative agents of cervical cancer [1]. The E6 and E7 early gene products are responsible for the oncogenic potential of high-risk HPVs [2]. The transforming activity of E6 and E7 is mainly mediated through the inactivation of cellular tumour suppressor proteins. High-risk HPV E6 is able to induce the functional inactivation and proteosomal degradation of p53 [3]. The E7 proteins of high-risk genital HPVs are able to bind to the retinoblastoma protein $(\mathrm{Rb})$ and to the related pocket proteins (p107 and p130), release E2F transcription factors from the pocket proteins, and induce the progression of the cell cycle [4-6]. The expression of the viral E6 and E7 proteins is indispensable for the normal replication cycle of both low-risk and high-risk HPV types [7].

Genital HPVs replicate in stratified squamous epithelial tissues, especially in the skin and mucous membranes of the genital area. The replication of the virus genome and the expression of viral genes are strictly linked to the differentiation of the keratinocyte host cell [ 8,9$]$. In the basal cells, low-level viral genome replication takes place, along with the expression of early genes, including the E6 and E7 oncogenes. High-level genome 
replication, expression of late genes and production of virions take place exclusively in well-differentiated epithelial layers [10].

The expression of HPV E6 and E7 oncogenes has been shown to induce a delay in the differentiation program of the host cell [11-13]. This is thought to have a role in the productive viral replication cycle, where the virus has to induce the cellular replication machinery in differentiating keratinocytes to provide replication of the viral genome [7, 9, 10]. Inhibition of cellular differentiation by high-risk HPVs is even more pronounced in premalignant and malignant genital lesions, such as cervical intraepithelial neoplasia (CIN) and cervical carcinoma. In these manifestations, a non-productive viral life cycle takes place, with a lack of late gene expression and the absence of virion production $[10,14]$.

Gene expression profiling has revealed that cervical cancer samples compared to normal cervical cells display reduced expression of several genes that are involved in keratinocyte differentiation, such as involucrin (IVL), small proline-rich proteins (SPRRs), S100A calcium-binding proteins and several different keratin (KRT) proteins [15, 16]. In accordance with this, studies using in vitro cell culture model systems (primary human keratinocytes transduced by recombinant retrovirus vectors) have revealed that the high-risk HPV proteins E6 and/or E7 are able to downregulate the expression of these differentiation-regulated genes [17-21]. However, there are few data on the mechanisms by which the HPV oncogenes can regulate the expression of keratinocyte differentiation genes. As these genes are known to be regulated mainly at the level of transcription [22], it is reasonable to assume that the HPV oncogenes might regulate the promoter activity of these cellular genes. Indeed, we showed in a recent study that HPV 16 E6 and E7 caused strong downregulation of the expression of involucrin (IVL) in human foreskin keratinocytes and found E6 to downregulate the activity of the IVL promoter [21].

In this study, we aimed to identify further keratinocyte differentiation genes that are regulated by the HPV oncogenes at the transcriptional level. We performed gene expression profiling in HPV-oncogene-transduced HFK cells and confirmed that HPV 16 E6 and E7 have the ability to downregulate the expression of several genes involved in keratinocyte differentiation. Luciferase reporter assays were performed to investigate the effects of HPV 16 E6 and E7 oncoproteins on the transcriptional activities of selected genes involved in keratinocyte differentiation. 


\section{Materials and methods}

\section{Cell culture}

Primary human foreskin keratinocytes (HFK) pooled from multiple neonatal foreskins were obtained from Invitrogen (Carlsbad, CA, USA). The culture and retroviral transduction of keratinocytes was described previously [21]. Briefly, HFK cells were cultured in Defined Keratinocyte-Serum Free Medium (DK-SFM, Invitrogen) and infected with culture supernatants from PA317 cell lines producing the control LXSN virus or LXSN-based retroviral vectors expressing HPV16 E6, HPV16 E7 or HPV16 E6/E7 genes.

\section{Microarray processing}

Total RNA was isolated from each transduced keratinocyte cell line in three biological replicates using an RNeasy Mini Kit (QIAGEN, Hilden, Germany). RNA integrity was checked on an Agilent Bioanalyzer 2100 (Agilent Technologies, Santa Clara, CA, USA). RNA samples with >9.0 RNA integrity number (RIN) values were used in the further experiments. A NanoDrop ND-1000 was used to determine RNA concentration. Global expression patterns were analyzed on Affymetrix GeneChip Human Gene 1.0 ST arrays (Affymetrix, Santa Clara, CA, USA). An Ambion WT Expression Kit (Ambion, Austin, TX, USA) and a GeneChip WT Terminal Labeling and Control Kit (Affymetrix) were used for amplifying and labeling $200 \mathrm{ng}$ of total RNA samples. Samples were hybridized at $45^{\circ} \mathrm{C}$ for 16 hours, and a standard washing protocol was then performed using an Affymetrix GeneChip Fluidics Station 450. The arrays were scanned on a GeneChip Scanner 7G (Affymetrix). RNA labelling and hybridization were carried out by UD-GenoMed Medical Genomic Technologies Ltd (Debrecen, Hungary).

\section{Microarray data analysis}

Microarray data were analyzed using GeneSpring 12 GX software (Agilent Technologies). Affymetrix CEL files were normalized using the Robust Multichip Analysis (RMA) algorithm and median normalization. Expressed genes were identified by filtering out the lowest 20 percentile of genes based on raw signal intensity. Then, genes that did not show at least a twofold difference compared to the median were also filtered out, and the remaining 3293 genes were called changing genes. Statistical analysis was performed on the list of changing genes using a one-way ANOVA test with Tukey's post hoc test. Differences with $p<0.05$ were considered statistically significant, and 
the fold change cutoff value was 1.5. Pathway analysis was performed using Ingenuity Pathway Analysis (IPA) software (Ingenuity Systems). The microarray data shown in this publication have been deposited in NCBI's Gene Expression Omnibus [23] and are accessible through GEO Series accession number GSE58841 (http://www.ncbi.nlm.nih.gov/geo/query /acc.cgi? acc $=$ gse58841).

\section{Quantitative real-time RT-PCR}

The reverse transcription and real-time PCR reactions from retrovirustransduced HFKs were performed as described previously [21]. The assay IDs of TaqMan Gene Expression Assays (Applied Biosystems, Foster City, CA, USA) are shown in the appropriate figures and tables. Glyceraldehyde 3-phosphate dehydrogenase (GAPDH; 0711024) was used as an endogenous control (Applied Biosystems). Each PCR reaction was performed in triplicate at least three times. The comparative $\mathrm{Ct}$ method was used to obtain the relative quantification (RQ) values with standard deviations (7500 System SDS Software, version 1.4).

\section{Plasmids}

The Renilla luciferase reporter vectors containing the promoter regions of KRT4 (S703756), KRT5 (S715429), SPRR1A (S703247), S100A8 (S703208) and DSC1 (S701128) genes were obtained from SwitchGear Genomics (Menlo Park, CA, USA). The pAP-1-Luc and pC/EBP-Luc plasmids containing several copies of binding sites for AP-1 and C/EBP transcription factors, respectively, were from Agilent Technologies. The pcDNA-based constructs expressing HPV16 E6 or E7 were described previously [24].

\section{Transient transfection and luciferase assay}

The luciferase reporter assay is a simple and sensitive tool for studying the transcriptional activities of viral or cellular gene promoters. Here, we used this method to study the effects of HPV oncoproteins on the promoter activities of cellular differentiation-associated genes in HFK cells cotransfected with luciferase reporter vectors along with expression vectors carrying the HPV oncogenes. Human foreskin keratinocytes (HFK) at passages 3-5 were plated on 6-well plates at approximately 70-80 \% confluence. The HFK cells were transfected with $0.5 \mu \mathrm{g}$ of reporter plasmids containing the promoter region of selected differentiation-associated genes (KRT4, KRT5, SPRR1A, S100A8 or DSC1) or reporter constructs containing 
multiple copies of AP-1 or C/EBP $\beta$ binding sites (pAP-1-Luc, pC/EBP-Luc), along with $0.25 \mu \mathrm{g}$ of expression vectors (pcDNA) encoding HPV 16 E6 and/or E7 genes using Effectene (QIAGEN) as described previously [21]. In brief, transfection mix was added to cells in Opti-MEM (Invitrogen) and incubated for $5 \mathrm{~h}$ at $37^{\circ} \mathrm{C}$, after which the medium was replaced by DK-SFM. Forty-eight hours after transfection, the cells were washed twice with PBS (phosphate-buffered saline) and lysed in Renilla Luciferase Assay Lysis Buffer or Cell Cycle Lysis Reagent (Promega, Madison, WI, USA). The luciferase activity of the cell extracts was measured using a Renilla Luciferase Assay System or Luciferase Assay System (Promega) and a Berthold luminometer. The Bradford protein assay was used to standardize for the protein concentration of the cell extracts. The provider of the luciferase reporter constructs (SwitchGear Genomics) did not recommend using co-transfected reporter plasmids to standardize for transfection efficiency. To analyse the results of luciferase tests, mean and SEM (standard error of mean) of standardized luciferase values were calculated. Each transfection experiment was performed independently at least three times. The statistical significance of the difference between mean values was evaluated using the ratio t-test (a paired t-test performed after logarithmic transformation of standardized luciferase values).

\section{Results}

\section{Global gene expression profiling of HFK cells transduced by HPV 16 oncogenes}

To explore the effects of HPV 16 E6 and E7 oncogenes on the global gene expression profile of primary HFK cells, microarray analyses were performed on low-passage HFK cells transduced by recombinant retroviruses.

Previously, we described the presence of HPV E6 and/or E7 mRNA in transduced cells and their effect on the level of the cellular p53 oncoprotein [21]. To further prove the presence of functionally active E6 and/or E7 proteins in the transduced cells, we first performed quantitative real-time RT-PCR analysis of the expression of two selected cellular genes that are known targets of E6 or E7 [25, 26]. As expected, HPV 16 E6 strongly induced the expression of the TERT (telomerase reverse transcriptase) gene, while E7 increased the level of CDC25A (cell division cycle 25A) mRNA in the transduced HFK cells (Fig. 1).

\section{Fig. 1}

The effect of HPV 16 E6, E7 oncogenes on the expression of CDC25A (A) and 
TERT (B) in human keratinocytes (HFK). Cells were transduced by retroviruses carrying either the control vector (LXSN) or constructs encoding HPV16 E6, E7 or E6/E7. Relative quantification (RQ) values were obtained by real-time RT-PCR analysis. The assay IDs of TaqMan gene expression assays for CDC25A and TERT were Hs00947994_m1, and Hs00972656_m1, respectively. The RQ value of cells transduced by LXSN was set to 1, and other values are shown relative to this. Each reaction was performed in triplicate at least three times. Representative graphs are shown, with error bars indicating standard deviation. $* *$ indicates significant $(\mathrm{p}<0.01)$ gene expression difference relative to LXSN

A

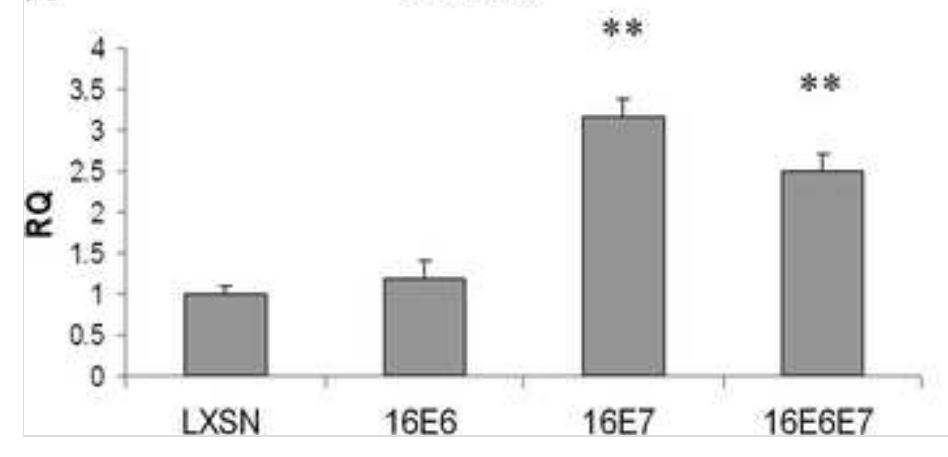

B

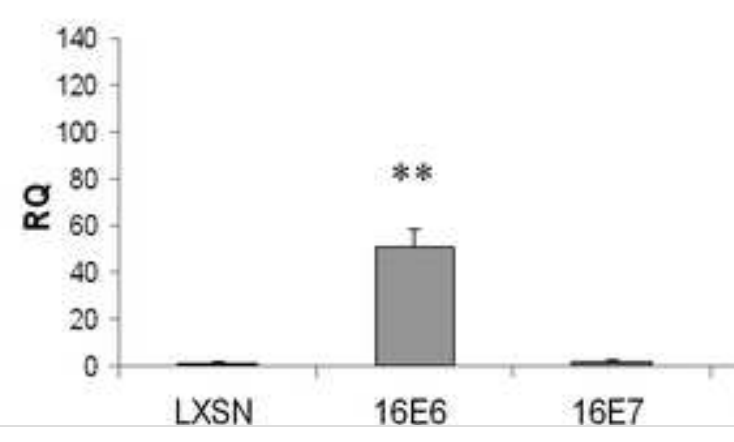

Using microarray analysis, we determined the global mRNA profile of three independent sets of cells transduced by virus vectors carrying HPV 16 E6, E7, or E6 and E7 genes compared to three sets of donor-matched cells transduced by LXSN control vector (12 samples in total). Genes that were differentially expressed under the four sets of conditions were identified by ANOVA test, and unsupervised hierarchical clustering was then performed. The generated heat map shows the separation between the conditions based on the ANOVA result (Supplementary Fig. 1). The list of genes whose expression was significantly altered by the HPV oncogenes (with a fold change of at least 1.5) is shown in Supplementary Table 1. IPA analysis revealed that these differentially expressed genes affect the regulation of cell cycle, cell death and proliferation pathways/functions (Table 1). In accordance with the results of previous studies using similar methodologies [17-20], we found that the expression of several genes that are known to be involved in keratinocyte differentiation (genes encoding involucrin (IVL), small proline-rich proteins (SPRRs), S100A calcium-binding proteins and several different keratin (KRT) proteins, among others) were significantly downregulated by the HPV oncoproteins (Tables 1 and 2). 


\section{Table 1}

Functional analysis of differentially expressed genes

\begin{tabular}{|c|c|c|c|}
\hline Function & p-value & $\begin{array}{l}\text { Number of } \\
\text { associated } \\
\text { genes }\end{array}$ & $\begin{array}{l}\text { Number of genes } \\
\text { associated with } \\
\text { keratinocyte } \\
\text { differentiation }\end{array}$ \\
\hline Cell cycle & $\begin{array}{l}2.22 \mathrm{E}-26 \text { to } \\
2.30 \mathrm{E}-03\end{array}$ & 142 & 2 \\
\hline $\begin{array}{l}\text { Cellular assembly } \\
\text { and organization }\end{array}$ & $\begin{array}{l}3.92 \mathrm{E}-22 \text { to } \\
2.07 \mathrm{E}-03\end{array}$ & 104 & 2 \\
\hline $\begin{array}{l}\text { DNA replication, } \\
\text { recombination, and } \\
\text { repair }\end{array}$ & $\begin{array}{l}3.92 \mathrm{E}-22 \text { to } \\
2.33 \mathrm{E}-03\end{array}$ & 130 & 0 \\
\hline $\begin{array}{l}\text { Cellular growth and } \\
\text { proliferation }\end{array}$ & $\begin{array}{l}1.49 \mathrm{E}-13 \text { to } \\
2.31 \mathrm{E}-03\end{array}$ & 174 & 7 \\
\hline $\begin{array}{l}\text { Cell death and } \\
\text { survival }\end{array}$ & $\begin{array}{l}1.18 \mathrm{E}-12 \text { to } \\
2.02 \mathrm{E}-03\end{array}$ & 176 & 5 \\
\hline
\end{tabular}

Table 2

Fold change values of keratinocyte differentiation genes whose expression was significantly altered by HPV16 E6, E7 or E6/E7 oncogenes in microarray analysis

\begin{tabular}{|c|c|c|c|c|c|}
\hline Gene symbol & Gene name & $16 \mathrm{E} 6^{\mathrm{a}}$ & $16 \mathrm{E} 7^{\mathrm{a}}$ & $16 \mathrm{E} 6 \mathrm{E} 7^{\mathrm{a}}$ & p-value \\
\hline DSC1 & Desmocollin 1 & -1.19 & -4.08 & -2.64 & 0.014 \\
\hline DSG1 & Desmoglein 1 & 1.32 & -2.44 & 1.21 & 0.021 \\
\hline FLG & Filaggrin & 1.19 & -2.39 & -1.56 & 0.040 \\
\hline IVL & Involucrin & -1.60 & -1.70 & -2.53 & 0.004 \\
\hline KRT1 & Keratin 1 & 1.04 & -2.93 & -1.50 & 0.035 \\
\hline KRT10 & Keratin 10 & 1.26 & -3.87 & 1.01 & 0.009 \\
\hline KRT13 & Keratin 13 & -1.25 & -2.29 & -1.70 & 0.031 \\
\hline KRT16 & Keratin 16 & -1.05 & -1.74 & 1.06 & 0.044 \\
\hline KRT19 & Keratin 19 & -1.56 & 1.26 & -1.90 & 0.012 \\
\hline
\end{tabular}

${ }^{\mathrm{a}}$ Fold change (FC) values are shown relative to vector-control-transduced cells (LXSN) 


\begin{tabular}{|c|c|c|c|c|c|}
\hline Gene symbol & Gene name & $16 \mathrm{E} 6^{\mathrm{a}}$ & $16 \mathrm{E} 7^{\mathrm{a}}$ & $16 \mathrm{E} 6 \mathrm{E} 7^{\mathrm{a}}$ & p-value \\
\hline LCE2B & $\begin{array}{l}\text { Late cornified } \\
\text { envelope } 2 \mathrm{~B}\end{array}$ & -1.01 & -1.75 & -1.21 & 0.011 \\
\hline S100A8 & $\begin{array}{l}\text { S100 calcium-binding } \\
\text { protein A } 8\end{array}$ & -1.17 & -1.26 & -2.11 & 0.030 \\
\hline S100A9 & $\begin{array}{l}\text { S100 calcium-binding } \\
\text { protein A9 }\end{array}$ & -1.37 & -1.43 & -3.31 & 0.005 \\
\hline SPRR1A & $\begin{array}{l}\text { Small proline-rich } \\
\text { protein } 1 \mathrm{~A}\end{array}$ & -1.15 & -1.55 & -1.97 & 0.021 \\
\hline SPRR1B & $\begin{array}{l}\text { Small proline-rich } \\
\text { protein } 1 \mathrm{~B}\end{array}$ & -1.11 & -1.25 & -1.51 & 0.039 \\
\hline SPRR2A|SPRR2B & $\begin{array}{l}\text { Small proline-rich } \\
\text { protein } 2 \mathrm{~A} \text { small } \\
\text { proline-rich protein } 2 \mathrm{~B}\end{array}$ & -1.87 & -1.39 & -2.81 & 0.031 \\
\hline SPRR3 & $\begin{array}{l}\text { Small proline-rich } \\
\text { protein } 3\end{array}$ & -1.97 & -4.06 & -3.79 & 0.014 \\
\hline
\end{tabular}

AQ1

\section{Effect of HPV 16 eneogenesoncoproteins on endogenous mRNA levels of selected keratinocyte differentiation genes}

To validate gene expression data obtained by microarray analysis, we performed quantitative real-time RT-PCR (qRT-PCR) assays on a subset of genes involved in keratinocyte differentiation. Considering that real-time PCR assays are known to have better sensitivity and reproducibility than microarray analysis, we included in the RT-PCR analysis certain genes that showed no change in expression in the microarray analysis but are known to have a role in keratinocyte differentiation (such as CNFN, KRT4, LOR, S100A10 and S100A12). As shown in Table 3, most of the genes analysed showed significantly decreased expression in the presence of the HPV 16 E6 and E7 oncogenes. Some of the genes investigated (such as KRT4 or S100A12) were found to have significantly reduced expression in the presence of the HPV oncoproteins in real-time RT-PCR analysis but not in microarray analysis. 


\section{Table 3}

Relative quantification values of selected keratinocyte differentiation genes obtained by quantitative real-time PCR analysis

\begin{tabular}{|c|c|c|c|c|}
\hline $\begin{array}{l}\text { Gene } \\
\text { symbol }\end{array}$ & Gene name & Assay ID ${ }^{a}$ & LXSN & $16 \mathrm{E} 6 \mathrm{E} 7^{\mathrm{b}}$ \\
\hline CNFN & Cornifelin & Hs00261196_m1 & $1 \pm 0.18$ & $0.46 \pm 0.03$ \\
\hline DSC1 & Desmocollin 1 & Hs00245189_m1 & $1 \pm 0.15$ & $0.17 \pm 0.01$ \\
\hline DSG1 & Desmoglein 1 & Hs00170047_m1 & $1 \pm 0.18$ & $0.63 \pm 0.03$ \\
\hline IVL & Involucrin & Hs00846307_s1 & $1 \pm 0.21$ & $0.13 \pm 0.02$ \\
\hline KRT1 & Keratin 1 & Hs00196158_m1 & $1 \pm 0.15$ & $0.23 \pm 0.01$ \\
\hline KRT4 & Keratin 4 & Hs00361611_m1 & $1 \pm 0.15$ & $0.09 \pm 0.01$ \\
\hline KRT5 & Keratin 5 & Hs00361185_m1 & $1 \pm 0.17$ & $1.85 \pm 0.18$ \\
\hline KRT10 & Keratin 10 & Hs00166289_m1 & $1 \pm 0.10$ & $0.72 \pm 0.04$ \\
\hline LOR & Loricrin & Hs01894962_s1 & $1 \pm 0.10$ & $0.80 \pm 0.03$ \\
\hline NOTCH1 & Notch homolog 1 & Hs01062014_m1 & $1 \pm 0.13$ & $0.50 \pm 0.03$ \\
\hline SERPINB2 & $\begin{array}{l}\text { Serpin peptidase } \\
\text { inhibitor, clade B } \\
\text { (ovalbumin), member } \\
2\end{array}$ & Hs00234032_m1 & $1 \pm 0.18$ & $0.49 \pm 0.02$ \\
\hline SPRR1A & $\begin{array}{l}\text { Small proline-rich } \\
\text { protein } 1 \mathrm{~A}\end{array}$ & Hs00954595_s1 & $1 \pm 0.13$ & $0.28 \pm 0.01$ \\
\hline SPRR1B & $\begin{array}{l}\text { Small proline-rich } \\
\text { protein } 1 \mathrm{~B}\end{array}$ & Hs00234164_m1 & $1 \pm 0.10$ & $0.37 \pm 0.03$ \\
\hline S100A8 & $\begin{array}{l}\text { S100 calcium-binding } \\
\text { protein A8 }\end{array}$ & Hs00374264_g1 & $1 \pm 0.12$ & $0.13 \pm 0.01$ \\
\hline S100A9 & $\begin{array}{l}\text { S100 calcium-binding } \\
\text { protein A9 }\end{array}$ & Hs00610058_m1 & $1 \pm 0.13$ & $0.11 \pm 0.01$ \\
\hline S100A10 & $\begin{array}{l}\text { S100 calcium-binding } \\
\text { protein A10 }\end{array}$ & Hs00741221_m1 & $1 \pm 0.12$ & $0.79 \pm 0.07$ \\
\hline S100A12 & $\begin{array}{l}\text { S100 calcium-binding } \\
\text { protein A } 12\end{array}$ & Hs00942835_g1 & $1 \pm 0.12$ & $0.10 \pm 0.01$ \\
\hline
\end{tabular}

${ }^{a}$ Assay IDs identify the TaqMan gene expression assays (Applied Biosystems) used for studying the gene expression changes of the indicated genes

${ }^{\mathrm{b}} \mathrm{RQ}$ (relative quantification) values (with standard deviation) are shown relative to vector-control-transduced cells (LXSN) 
Seven of the genes with altered expression in the presence of the HPV oncoproteins (in microarray analysis and/or real-time RT-PCR assays) were chosen for detailed gene expression analysis by real-time RT-PCR assays (Fig. 2). For comparison, a basal keratin gene whose expression is known not to be altered by differentiation (KRT5) was also included in the analysis. As expected, KRT5 mRNA levels were not decreased by the HPV oncoproteins. Rather, the HPV oncoproteins caused a 1.5- to 2-fold increase in the expression of KRT5. On the other hand, the keratinocyte differentiation genes analysed by real-time RT-PCR (DSC1, KRT1, KRT4, KRT10, S100A8, S100A9 and SPRR1A) usually showed significantly decreased expression in the presence of the HPV oncoproteins (Fig. 2). In a previous study, we also found the expression of the human IVL (involucrin) gene to be downregulated by HPV 16 oncoproteins [21]. Taken together, our results indicate that it may be a general function of the HPV 16 E6 and E7 oncoproteins to downregulate the expression of genes involved in the differentiation of stratified squamous epithelial cells.

\section{Fig. 2}

Effect of HPV 16 oncogenes on the endogenous mRNA level of DSC1 (A), KRT1 (B), KRT4 (C), KRT10 (D), S100A8 (E), S100A9 (F), SPRR1A (G) and KRT5 (H) in HFK cells. Generation of cell lines and estimation of RQ values were performed as described in the legend to Fig. 1. Each reaction was performed in triplicate at least three times. Representative graphs are shown, with error bars indicating standard deviation. Stars indicate significant gene expression differences relative to LXSN $(* \mathrm{p}<0.05$, ** $\mathrm{p}<0.01$, ***p $<0.001)$ 

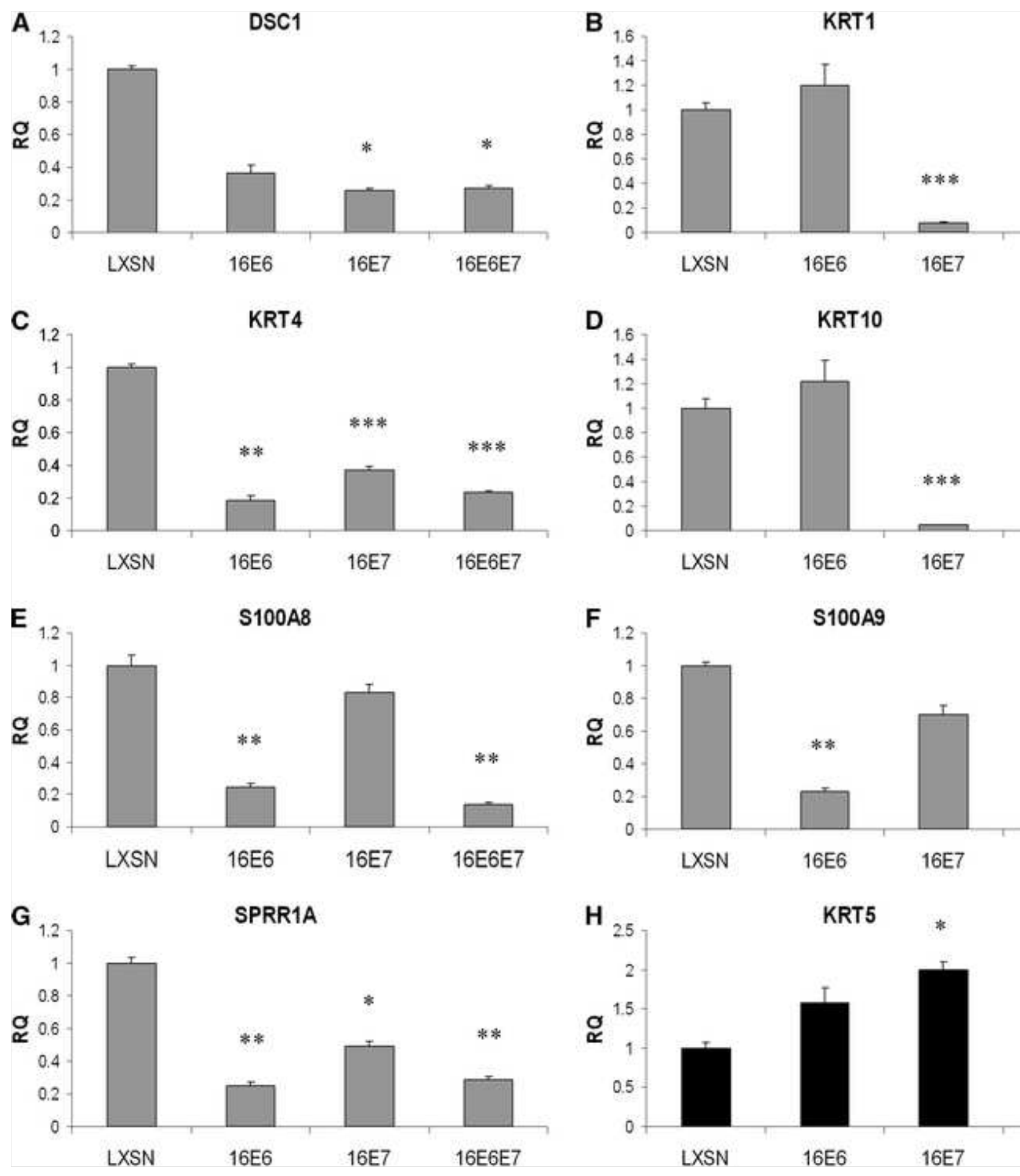

Effect of HPV 16 eneegenesoncoproteins on the promoter activities of selected keratinocyte differentiation genes

To see the effects of the HPV oncogenes on the transcriptional activities of keratinocyte differentiation genes whose expression has been analysed by real-time RT-PCR assays, luciferase reporter assays were performed. These experiments were conducted in HFK cells that were transiently co-transfected with reporter vectors containing the promoters of different keratinocyte differentiation genes (DSC1, KRT4, S100A8 and SPRR1A) along with HPV 16 E6, E7 or E6 and E7 expression vectors (Fig. 3). The promoter activities 
of each of these four genes were significantly decreased in cells transfected with HPV 16 E6 and E7 oncogenes together (16E6E7) compared to cells transfected with a control expression vector. As expected, the HPV oncoproteins had little if any effect on the transcriptional activity of the KRT5 promoter (Fig. 3E). Our results suggest that the downregulation of expression by the HPV 16 oncoproteins of several genes involved in keratinocyte differentiation is caused at least partly by inhibition of transcriptional activities of these genes.

\section{Fig. 3}

Promoter activity of DSC1 (A), KRT4 (B), S100A8 (C), SPRR1A (D) and KRT5 (E) in the presence of HPV 16 E6, E7 or both in human keratinocytes. HFK cells were co-transfected with reporter plasmids containing the promoters of the investigated differentiation-associated genes, along with either control vector (pcDNA) or expression constructs encoding HPV16 E6, E7 or both as indicated. The Renilla luciferase activities are shown relative to the activity of cells transfected with reporter plasmids and the empty expression vector (pcDNA). Data are from at least three independent experiments, with standard errors shown as error bars. Stars indicate the significance of difference in transcriptional activity compared to control vector transfected cells $(* \mathrm{p}<0.05$, $* * \mathrm{p}<0.01, * * * \mathrm{p}<0.001)$ 

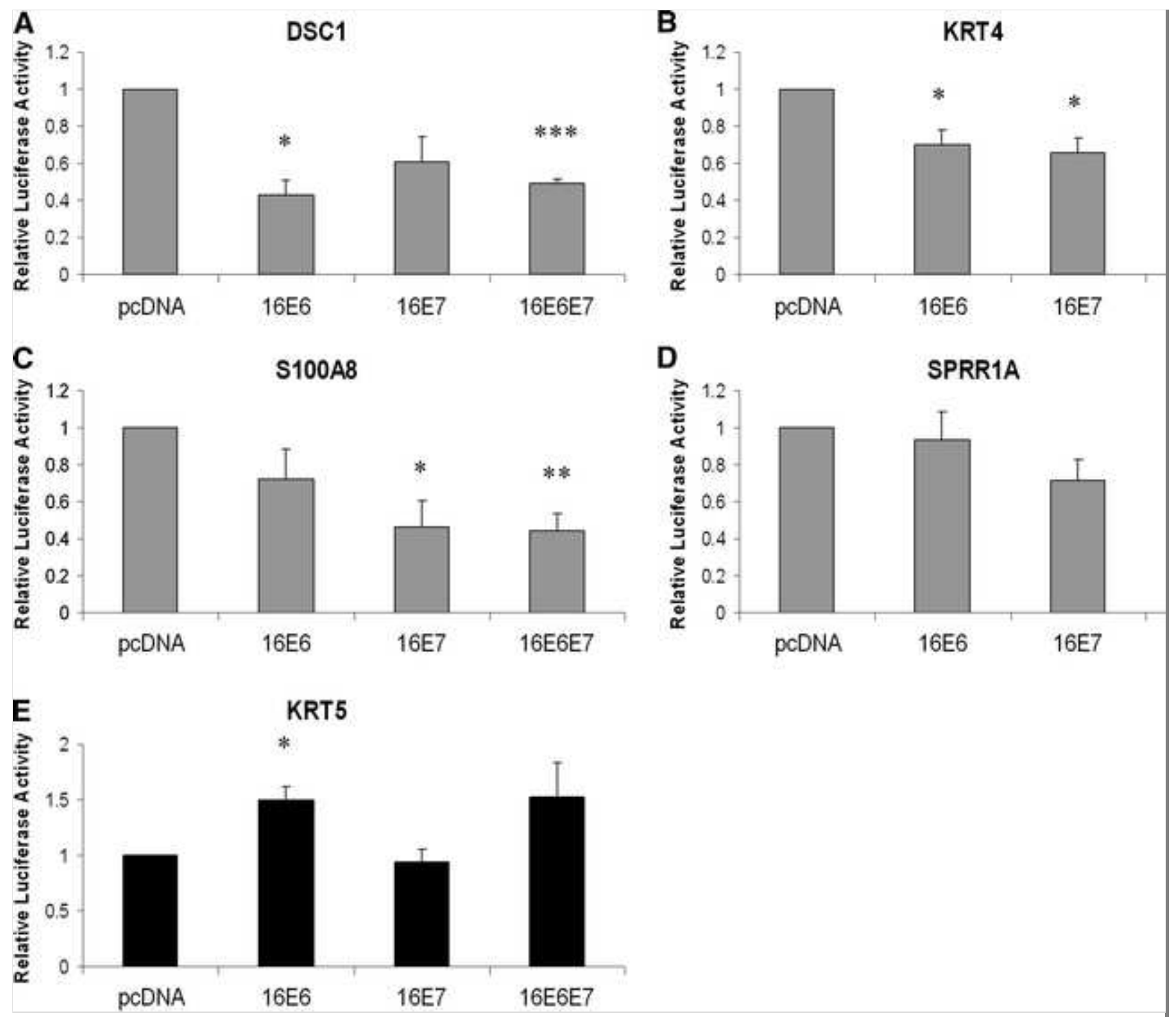

\section{Effect of HPV 16 encegenesoncoproteins on the activities of AP-1 and C/EBP transcription factors}

We presumed that the HPV 16 oncoproteins downregulate the expression of keratinocyte differentiation genes by modifying the activities of certain transcription factors. Sequence analysis of the promoters that were shown here to be inhibited by the HPV oncoproteins revealed that each contains putative binding sites for AP-1 (activator protein 1) and C/EBP (CCAAT enhancer binding protein) transcription factor family members (Fig. 4). Therefore, we decided to explore if the HPV 16 oncogenes have any effect on the activities of these transcription factors. To this end, HFK cells were transfected with luciferase reporter vectors containing multiple copies of binding sites for the transcription factors AP-1 (jun/fos) or C/EBP along with HPV E6/E7 expression vectors. The results of luciferase tests showed that the HPV 16 E6 and E7 oncogenes together had a significant downregulating effect on both C/EBP and AP-1 activity (Fig. 5). These results suggest that the 
downregulating effect of HPV 16 oncogenes on the promoters of certain keratinocyte differentiation genes may be caused by inhibiting the activities of transcription factors that are key regulators of these promoters, such as C/EBP and AP-1 family members.

\section{Fig. 4}

Schematic representation of SwitchGear Genomics reporter constructs containing DSC1, S100A8, SPRR1A and KRT4 promoters in front of the Renilla luciferase gene. Selected transcription factor recognition sites are indicated according to TFSEARCH (www.cbrc.jp/research /db/TFSEARCH.html) and the JASPAR database (jaspar.genereg.net) at threshold score 80.0 (C/EBP-CCAAT enhancer binding protein)

\section{DSC1}

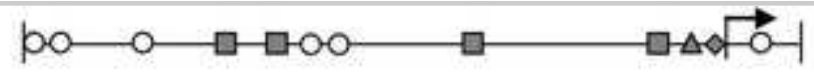

S100A8

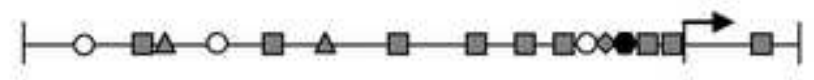

SPRR1A

KRT4

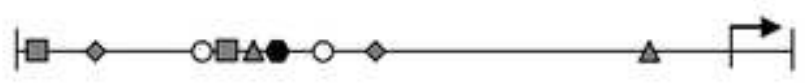
$\triangle$ - putative AP1 factor binding site
$\square$ - putative C/EBP factor binding site
0 - putative Ets factor binding site
- putative POU domain binding site
$\diamond$ - putative Sp1 factor binding site
- Transcription start site

\section{Fig. 5}

The effect of HPV oncogenes on the transcriptional activity of the C/EBP (A) and AP-1 (B) reporters in HFK cells. Human keratinocytes were transfected with the reporter constructs, along with either control vector (pcDNA) or constructs expressing HPV16 E6, E7 or both as indicated. The luciferase activity of cells co-transfected with reporter constructs and the empty expression vector (pcDNA) was set to 1 , and the activities in other transfections are relative to this. Results are from at least three independent experiments, with standard errors shown as error bars. Stars indicate the significance of difference in transcriptional activity compared to control-vector-transfected cells $(* \mathrm{p}<0.05, * * \mathrm{p}<0.01)$ 


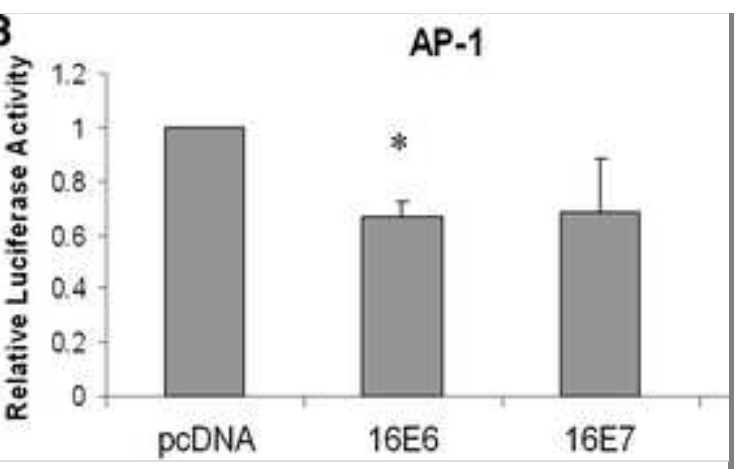

\section{Discussion}

In this study, we explored the effects of HPV 16 oncogenes on the expression of cellular genes involved in keratinocyte differentiation by using microarray analysis and quantitative real-time PCR assays. Microarray analysis is a valuable method of global gene expression profiling that is capable of measuring the expression levels of thousands of cellular genes. However, this method has been shown to have relatively poor performance in demonstrating expression changes of genes with low mRNA levels [27]. Also in this study, some of the genes with altered expression by the HPV oncogenes (such as CNFN, KRT4, LOR, S100A10 and S100A12) could be identified by real-time PCR assays, but not by microarray analysis.

Most of the keratinocyte differentiation genes found here to be downregulated by the HPV 16 oncogenes have already been shown to have reduced expression in cervical cancer compared to normal cervical cells [15, 16]. Accordingly, these genes have been shown to be downregulated by high-risk HPV oncogenes by using in vitro cell culture model systems [17-21]. Thus, it seems to be a general activity of high-risk HPV oncoproteins to downregulate the expression of genes associated with keratinocyte differentiation. This activity of HPV oncoproteins may have a role in the productive viral life cycle [9]. The delay of host cell differentiation is also a prominent feature of HPV-associated premalignant cervical lesions (CIN), and it can be also seen in HPV-immortalized keratinocytes grown in organotypic (raft) culture [28-30].

There are few data on the mechanism of action of the HPV oncogenes on the expression of keratinocyte differentiation genes. In a previous study, we showed that HPV 16 E6 downregulates the activity of the human IVL promoter [21]. In this study, we went on to see the effects of HPV 16 oncogenes on the transcriptional activities of further differentiation-associated genes (DSC1, KRT4, S100A8 and SPRR1A), which were shown to have 
reduced expression by the HPV oncoproteins in this study and also in previous reports [17-20]. We found that the promoters of each of these genes were usually downregulated by the HPV oncogenes. In each case, the strongest effect was found when both HPV oncoproteins were expressed. It may be speculated that the high-risk HPV E6 and E7 oncoproteins have an additive or synergistic effect on the expression of keratinocyte differentiation genes.

Usually, there was good correlation between the gene expression data obtained by real-time RT-PCR and the promoter activity data obtained by luciferase tests (compare Fig. 2 and 3 ). However, there were also examples where the promoter activities did not correspond closely to the gene expression data. For instance, HPV 16 E7 had no significant effect on the S100A8 mRNA level (Fig. 2E), while it had a significant downregulating activity on S100A8 promoter activity (Fig. 3C). On the other hand, HPV 16 E6 strongly decreased the SPRR1A mRNA level (Fig. 2G), while it had no significant effect on SPRR1A promoter activity (Fig. 3D). These results may indicate that, in addition to transcriptional regulation, other mechanisms (such as post-transcriptional regulation) may contribute to the downregulation of expression of these genes by the HPV oncoproteins. HPV 16 E6 has been reported to induce the expression of the hTERT gene at the level of transcription [3]. In addition, a cellular factor (NFX1-123) has been shown to increase hTERT mRNA stability in the presence of HPV 16 E6. Thus, transcriptional and posttranscriptional mechanisms seem to co-operate in the regulation of expression of hTERT by HPV 16 E6. It is also conceivable that, in addition to transcriptional regulation, posttranscriptional mechanisms (such as regulation of RNA processing, transport or mRNA stability) also contribute to the downregulation of expression of keratinocyte differentiation genes by the HPV oncoproteins.

The HPV oncoproteins had no inhibitory effect on the promoter of KRT5, which is a basal keratin gene, i.e., its expression is not regulated by differentiation. Thus, it appears that the HPV 16 oncoproteins specifically downregulate the transcription of keratinocyte genes whose expression is regulated by differentiation. Taken together, our luciferase results indicate that the HPV 16 oncogenes have a downregulating effect on the transcriptional activities of several keratinocyte differentiation genes, and this mechanism seems to contribute to the reduced expression of these genes in premalignant and malignant cervical lesions compared to normal cervical epithelium [ 15 , 
$16]$.

We found that each of the promoters that were shown in this study to be downregulated by the HPV oncoproteins have putative binding sites for AP-1 (jun/fos) and C/EBP transcription factors (Fig. 4). These transcription factors, among others, have been shown to be significant in the transcriptional regulation of keratinocyte differentiation genes and are themselves regulated by epithelial differentiation [22, 31-35]. Our experiments performed with reporter constructs containing binding sites for these transcription factors indicated that the HPV oncoproteins had significant inhibitory effects on the activities of these transcription factors. Therefore, it is conceivable that the HPV 16 oncoproteins downregulate certain keratinocyte differentiation promoters by modifying the activities of AP-1 and C/EBP transcription factors. In order to prove this scenario, further experiments would be needed, including promoter mutagenesis and chromatin immunoprecipitation (ChIP) assays.

In summary, gene expression analysis performed in this study in HPV-oncogene-transduced HFK cells confirmed that HPV 16 E6 and E7 oncogenes downregulate the expression of several genes involved in keratinocyte differentiation. Furthermore, luciferase reporter assays revealed that the HPV 16 E6 and E7 oncoproteins were able to downregulate the promoter activity of several of these genes. Our results suggest that the HPV 16 E6 and/or E7 oncogenes may have the potential to downregulate the expression of several keratinocyte differentiation genes, at least partially by downregulating their promoter activity. This activity of the HPV oncoproteins may have a role in the productive virus life cycle, and also in virus-induced carcinogenesis.

\section{Acknowledgments}

The research of EG was supported by the European Union and the State of Hungary, co-financed by the European Social Found in the framework of TÁMOP 4.2.4.A/2-11-1-2012-0001 'National Excellence Program'. This study was supported by a grant from the Hungarian Scientific Research Fund (OTKA K 81422).

\section{Electronic supplementary material}

Below is the link to the electronic supplementary material. 
Supplementary material 1 (PDF $75 \mathrm{~kb}$ )

Supplementary material 2 (PDF $176 \mathrm{~kb})$

\section{References}

1. McLaughlin-Drubin ME, Munger K (2009) Oncogenic activities of human papillomaviruses. Virus Res 143:195-208

2. Ghittoni R, Accardi R, Hasan U, Gheit T, Sylla B, Tommasino M (2010) The biological properties of E6 and E7 oncoproteins from human papillomaviruses. Virus Genes 40:1-13

3. Howie HL, Katzenellenbogen RA, Galloway DA (2009) Papillomavirus E6 proteins. Virology 384:324-334

4. McLaughlin-Drubin ME, Munger K (2009) The human papillomavirus E7 oncoprotein. Virology 384:335-344

5. Banerjee NS, Genovese NJ, Noya F, Chien WM, Broker TR, Chow LT (2006) Conditionally activated E7 proteins of high-risk and low-risk human papillomaviruses induce $S$ phase in postmitotic, differentiated human keratinocytes. J Virol 80:6517-6524

6. Cheng S, Schmidt-Grimminger DC, Murant T, Broker TR, Chow LT (1995) Differentiation-dependent up-regulation of the human papillomavirus E7 gene reactivates cellular DNA replication in suprabasal differentiated keratinocytes. Genes Dev 9:2335-2349

7. Van Doorslaer K, Burk RD (2010) Evolution of human papillomavirus carcinogenicity. Adv Virus Res 77:41-62

8. Longworth MS, Laimins LA (2004) Pathogenesis of human papillomaviruses in differentiating epithelia. Microbiol Mol Biol Rev 68:362-372

9. Kajitani N, Satsuka A, Kawate A, Sakai H (2012) Productive lifecycle 
of human papillomaviruses that depends upon squamous epithelial differentiation. Front Microbiol 3:152

10. Doorbar J, Quint W, Banks L, Bravo IG, Stoler M, Broker TR, Stanley MA (2012) The biology and life-cycle of human papillomaviruses. Vaccine 30(Suppl 5):F55-F70

11. Pei XF, Sherman L, Sun YH, Schlegel R (1998) HPV-16 E7 protein bypasses keratinocyte growth inhibition by serum and calcium. Carcinogenesis 19:1481-1486

12. Sherman L, Schlegel R (1996) Serum- and calcium-induced differentiation of human keratinocytes is inhibited by the E6 oncoprotein of human papillomavirus type 16. J Virol 70:3269-3279

13. Zehbe I, Richard C, DeCarlo CA, Shai A, Lambert PF, Lichtig H, Tommasino M, Sherman L (2009) Human papillomavirus 16 E6 variants differ in their dysregulation of human keratinocyte differentiation and apoptosis. Virology 383:69-77

14. Doorbar J (2005) The papillomavirus life cycle. J Clin Virol 32(Suppl 1):S7-S15

15. Santin AD, Zhan F, Bignotti E, Siegel ER, Cane S, Bellone S, Palmieri M, Anfossi S, Thomas M, Burnett A, Kay HH, Roman JJ, O’Brien TJ, Tian E, Cannon MJ, Shaughnessy J Jr, Pecorelli S (2005) Gene expression profiles of primary HPV16- and HPV18-infected early stage cervical cancers and normal cervical epithelium: identification of novel candidate molecular markers for cervical cancer diagnosis and therapy. Virology 331:269-291

16. Wong YF, Cheung TH, Tsao GS, Lo KW, Yim SF, Wang VW, Heung MM, Chan SC, Chan LK, Ho TW, Wong KW, Li C, Guo Y, Chung TK, Smith DI (2006) Genome-wide gene expression profiling of cervical cancer in Hong Kong women by oligonucleotide microarray. Int J Cancer 118:2461-2469

17. Duffy CL, Phillips SL, Klingelhutz AJ (2003) Microarray analysis identifies differentiation-associated genes regulated by human papillomavirus type 16 E6. Virology 314:196-205 
18. Karstensen B, Poppelreuther S, Bonin M, Walter M, Iftner T, Stubenrauch F (2006) Gene expression profiles reveal an upregulation of E2F and downregulation of interferon targets by HPV18 but no changes between keratinocytes with integrated or episomal viral genomes. Virology 353:200-209

19. Wan F, Miao X, Quraishi I, Kennedy V, Creek KE, Pirisi L (2008) Gene expression changes during HPV-mediated carcinogenesis: a comparison between an in vitro cell model and cervical cancer. Int $\mathbf{J}$ Cancer 123:32-40

20. Kravchenko-Balasha N, Mizrachy-Schwartz S, Klein S, Levitzki A (2009) Shift from apoptotic to necrotic cell death during human papillomavirus-induced transformation of keratinocytes. J Biol Chem 284:11717-11727

21. Gyöngyösi E, Szalmás A, Ferenczi A, Kónya J, Gergely L, Veress G (2012) Effects of human papillomavirus (HPV) type 16 oncoproteins on the expression of involucrin in human keratinocytes. Virol J 9:36

22. Eckert RL, Crish JF, Banks EB, Welter JF (1997) The epidermis: genes on-genes off. J Invest Dermatol 109:501-509

23. Edgar R, Domrachev M, Lash AE (2002) Gene Expression Omnibus: NCBI gene expression and hybridization array data repository. Nucleic Acids Res 30:207-210

24. Murvai M, Borbély AA, Kónya J, Gergely L, Veress G (2004) Effect of human papillomavirus type 16 E6 and E7 oncogenes on the activity of the transforming growth factor-beta2 (TGF-beta2) promoter. Arch Virol 149:2379-2392

25. Liu X, Roberts J, Dakic A, Zhang Y, Schlegel R (2008) HPV E7 contributes to the telomerase activity of immortalized and tumorigenic cells and augments E6-induced hTERT promoter function. Virology 375:611-623

26. Katich SC, Zerfass-Thome K, Hoffmann I (2001) Regulation of the Cdc25A gene by the human papillomavirus Type 16 E7 oncogene.

Oncogene 20:543-550 
27. Beckman KB, Lee KY, Golden T, Melov S (2004) Gene expression profiling in mitochondrial disease: assessment of microarray accuracy by high-throughput Q-PCR. Mitochondrion 4:453-470

28. McCance DJ, Kopan R, Fuchs E, Laimins LA (1988) Human papillomavirus type 16 alters human epithelial cell differentiation in vitro. Proc Natl Acad Sci USA 85:7169-7173

29. Blanton RA, Perez-Reyes N, Merrick DT, McDougall JK (1991) Epithelial cells immortalized by human papillomaviruses have premalignant characteristics in organotypic culture. Am J Pathol 138:673-685

30. Merrick DT, Blanton RA, Gown AM, McDougall JK (1992) Altered expression of proliferation and differentiation markers in human papillomavirus 16 and 18 immortalized epithelial cells grown in organotypic culture. Am J Pathol 140:167-177

31. Eckert RL, Crish JF, Efimova T, Dashti SR, Deucher A, Bone F, Adhikary G, Huang G, Gopalakrishnan R, Balasubramanian S (2004) Regulation of involucrin gene expression. J Invest Dermatol 123:13-22

32. Mehic D, Bakiri L, Ghannadan M, Wagner EF, Tschachler E (2005) Fos and jun proteins are specifically expressed during differentiation of human keratinocytes. J Invest Dermatol 124:212-220

33. Rossi A, Jang SI, Ceci R, Steinert PM, Markova NG (1998) Effect of AP-1 transcription factors on the regulation of transcription in normal human epidermal keratinocytes. J Invest Dermatol 110:34-40

34. Zhu S, Oh HS, Shim M, Sterneck E, Johnson PF, Smart RC (1999) $\mathrm{C} / \mathrm{EBPbeta}$ modulates the early events of keratinocyte differentiation involving growth arrest and keratin 1 and keratin 10 expression. Mol Cell Biol 19:7181-7190

35. Rozenberg JM, Bhattacharya P, Chatterjee R, Glass K, Vinson C (2013) Combinatorial recruitment of CREB, C/EBPbeta and c-Jun determines activation of promoters upon keratinocyte differentiation. PLoS ONE 8:e78179 